\title{
Monitoring of epitaxial graphene anodization
}

Mikhail Vagin, Alina Sekretareva, Ivan Gueorguiev Ivanov, Anna Håkansson, Tihomir Iakimov, Mikael Syväjärvi, Rositsa Yakimova, Ingemar Lundström and Mats Eriksson

The self-archived version of this journal article is available at Linköping University Electronic Press:

http:// urn.kb.se/ resolve?urn=urn:nbn:se:liu:diva- 138227

N.B.: When citing this work, cite the original publication.

Vagin, M., Sekretareva, A., Gueorguiev Ivanov, I., Håkansson, A., Iakimov, T., Syväjärvi, M., Yakimova, R., Lundström, I., Eriksson, M., (2017), Monitoring of epitaxial graphene anodization, Electrochimica Acta, 238, 91-98. https:// dx.doi.org/ 10.1016/j.electacta.2017.04.016

Original publication available at:

https:// dx.doi.org/ 10.1016/j.electacta.2017.04.016

Copyright: Elsevier

http:// www.elsevier.com/

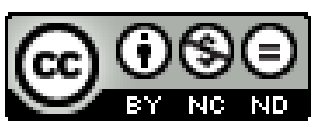




\section{Monitoring of epitaxial graphene anodization}

Mikhail Yu. Vagin ${ }^{1,2 *}$, Alina N. Sekretaryova ${ }^{1,3}$, Ivan G. Ivanov ${ }^{1}$, Anna Håkansson ${ }^{1,2}$, Tihomir Iakimov $^{1,4}$, Mikael Syväjärvi ${ }^{1,4}$, Rositsa Yakimova ${ }^{1,4}$, Ingemar Lundström ${ }^{1}$, Mats Eriksson ${ }^{1}$

${ }^{1}$ Department of Physics, Chemistry and Biology, Linköping University, SE-581 83, Linköping, Sweden; e-mail:mikva@ifm.liu.se

${ }^{2}$ Laboratory of Organic Electronics, Department of Science and Technology, Linköping University, SE-601 74, Norrköping, Sweden

${ }^{3}$ Department of Chemistry, Stanford University, Stanford, CA 94305-5080, USA

${ }^{4}$ Graphensic AB, Teknikringen 1F, SE-58330 Linköping, Sweden. 


\section{Abstract}

Anodization of a graphene monolayer on silicon carbide was monitored with electrochemical impedance spectroscopy. Structural and functional changes of the material were observed by Raman spectroscopy and voltammetry. A 21 fold increase of the specific capacitance of graphene was observed during the anodization. An electrochemical kinetic study of the $\mathrm{Fe}(\mathrm{CN}) 6^{3-/ 4-}$ redox couple showed a slow irreversible redox process at the pristine graphene, but after anodization the reaction rate increased by several orders of magnitude. On the other hand, the $\mathrm{Ru}\left(\mathrm{NH}_{3}\right)_{6}{ }^{3+/ 2+}$ redox couple proved to be insensitive to the activation process. The results of the electron transfer kinetics correlate well with capacitance measurements. The Raman mapping results suggest that the increased specific capacitance of the anodized sample is likely due to a substantial increase of electron doping, induced by defect formation, in the monolayer upon anodization. The doping concentration increased from less than $1 \times 10^{13}$ of the pristine graphene to $4-8 \times 10^{13}$ of the anodized graphene.

Keywords: epitaxial graphene, graphene monolayer, anodization, electrode kinetics, Raman spectroscopy, voltammetry, electrochemical impedance spectroscopy

\section{Introduction}

Graphene is a nanomaterial making a significant impact in a variety of applications [1-5] as well as in fundamental science because it offers the possibility to study the processes on the graphitic materials at the simplest level of structural organization.

Due to the planar conjugation of the $\mathrm{sp}^{2}$ bonds in graphene, there is a significant difference of the density of electronic states (DOS) at in-plane and out-of-plane conduction. This determines two distinct structural contributions (basal and edge plane respectively) to the behavior of all graphitic 
materials yielding an electrochemical anisotropy [6, 7]. The majority of recent electrochemical studies of graphene used bulk materials based on graphene flakes possessing a large contribution of edges with high DOS [8], which led to a confusion about the graphene electrochemical activity. Flat graphene sheets with controllable exposure of the basal plane are available for electrochemical measurements [9] via growth by chemical vapor deposition [10-12] or via thermal annealing [1316] of crystalline structures of silicon carbide yielding epitaxial graphene (EG). The annealing in an inert atmosphere yields a slow formation of high quality graphene monolayer on the solid semiinsulating support [17].

By introducing defects in the graphene lattice, an increased electrochemical activity can be obtained. Chemical modification of graphenes leads to modulation of their properties [18]. Anodization, a technique to induce defects into highly oriented pyrolytic graphite (HOPG) [19], is an electrochemical approach to create defects also in the graphene lattice. The anodization initially generates hydroxylic and carboxylic groups, which for prolonged anodization causes strain in the graphene lattice resulting in fracturing [15]. Anodization at high potentials may remove contaminants from the surface hindering the kinetics of surface-sensitive redox processes [20] yielding anodized EG as an advanced sensing material [13, 15].

Here we report on monitoring of the anodization process, which is a key step of EG activation. The changes in capacitive, reactive and structural properties of the graphene have been comparatively studied with Raman spectroscopy and electrochemical methods at conditions of electrocapacitive and redox processes.

\section{Experimental}

\subsection{Reagents}

All inorganic salts were purchased from Sigma (Sweden) and used as received. Experiments were carried out with Milli-Q water from a Millipore Milli-Q system. 


\subsection{Samples and processes}

An Autolab type III potentiostat (Autolab, EcoChemie, Netherlands) was used for the electrochemical measurements. An Ag/AgCl electrode in $3 \mathrm{M} \mathrm{KCl}$ and a platinum wire were used as reference electrode and counter electrode, respectively, for all measurements.

The samples of nominally monolayer epitaxial graphene on SiC (substrate area $7 \times 7 \mathrm{~mm}^{2}$ and thickness $0.4 \mathrm{~mm}$ ) and the electrochemical cell employed for the measurements have been obtained from Graphensic AB. Graphene was grown on the Si-face of the SiC substrates which typically yields a continuous coverage of more than $90 \%$ monolayer graphene while the rest is small patches of bilayer graphene [21]. The cell consisted of a cup of $300 \mu \mathrm{l}$ with a $2 \mathrm{~mm}$ diameter hole with a Vitron o-ring disk on the bottom. The EG sample was fixed under the hole with the oring using screws on the lid. A dry contact to the EG was formed by an aluminum adhesive. The mounted sample was kept inside the cell during all electrochemical measurements in order to avoid sample drift.

The EG electrode anodization was carried out in a $0.1 \mathrm{M} \mathrm{KNO}_{3}$ solution in a continuous pulsed mode with a 0.5 seconds oxidation pulse at $2 \mathrm{~V}$ followed by a 0.125 seconds reduction pulse at 0.1 $\mathrm{V}[19,22]$.

\subsection{Raman spectroscopy}

The properties of the sample before and after anodization were also investigated using microRaman spectroscopy. A lens with 100X magnification and a solid-state laser at $532 \mathrm{~nm}$ were employed in these measurements. The scattered light was analyzed using a monochromator (JobinYvon, model HR460) equipped with a CCD camera. The spectral resolution of the system is 5.5 $\mathrm{cm}^{-1}$. The monolayer and bilayer regions of EG on the sample were distinguished using the reflectance from the sample recorded simultaneously with the Raman spectra [23]. The laser power at the sample was $\sim 17 \mathrm{~mW}$ focused to a spot with a diameter of approximately $0.85 \mu \mathrm{m}$. 


\subsection{Atomic force microscopy}

Atomic force microscopy has been done by Dimension 3100SPM (Veeco Instruments) equipped with acoustic/vibration isolation system and the VT-102 vibration isolation table in tapping mode using Micromasch NSC14 cantilevers.

\section{Results and discussion}

\subsection{Anodization monitoring}

The impedance spectra acquired for pristine and short-time anodized EG in a pure aqueous electrolyte showed large parts of a well-defined semicircle in a complex plane plot of the frequency-normalized admittance (Fig. 1A) from which the double layer capacitance on the EG surface can be extracted without the use of model-dependent fitting. The electrodes of pristine EG and EG anodized for $15 \mathrm{~s}$ and $30 \mathrm{~s}$ showed values of the double layer capacitance of the electrode|solution interface of $1.04 \mu \mathrm{F}, 1.66 \mu \mathrm{F}$ and $2.68 \mu \mathrm{F}$, respectively. This corresponds to values of the specific capacitance of 33.1, 52.9 and $85.4 \mu \mathrm{F} \mathrm{cm}^{-2}$ for pristine and anodized EG for $15 \mathrm{~s}$ and $30 \mathrm{~s}$, respectively, which is more than ten times larger than the value obtained for pristine EG from previous voltammetry measurements [15], which probably illustrates the different conditions of growth via thermal annealing.

The spectra obtained at longer time of anodization revealed more complex impedance patterns (Fig. 1SA), which were analyzed with equivalent circuits. The presence of two time-resolved processes visible in the Bode plot (Fig. 1SB) was modelled by two RC elements in the simplest unified equivalent circuit (Inset in Fig. 1B) developed for an electrode covered with a damaged coating $[24,25]$ and utilized for the fitting of the spectra of the anodized EG electrode. During the course of anodization the EG coating is partly damaged. Therefore, the electrode-solution interface can be divided into two physical regions: (1) an electric double layer formed in the solution part of the interface and (2) a region related to the structural changes of the EG layer during anodization. 
Since the boundary between the layers is not ideally smooth, due to increased surface roughness with anodization time, a quantitative analysis of the electrode impedance response requires a more complicated, distributed circuit model featuring constant phase elements (CPE) rather than pure capacitors. The capacitance values have been calculated as [26]:

$C=\left(P\left(R_{S}\right)^{(1-\phi)}\right)^{1 / \phi}$

where $R_{S}$ is the solution resistance, $P$ is a fitting parameter of the CPE and $\phi$ is the fitted exponent factor, which varies from 0 to 1 . When $\phi$ is tending to 0 the CPE behaves as a pure resistor and when $\phi=1$ the CPE represents a pure capacitor. The equivalent circuit providing the best fit consists of the solution resistance $R_{S}$ and two combined R-CPE units (I and II). A single set of parameters has been used to simultaneously fit the real and imaginary parts of the impedance over the frequency range from $0.1 \mathrm{~Hz}$ to $5 \mathrm{kHz}$. A value of the fitting quality parameter $\chi^{2}$ of $\leq 0.001$ obtained for all spectra indicates a very good fit.

The dynamics of the changes of the fitted impedance parameters with anodization time showed a complex behavior and the interpretation of the data is therefore not straightforward. A plausible interpretation of the data is, however, attempted in the following. The increase of the solution resistance (Fig. 1C) appearing after $60 \mathrm{~s}$ of anodization might illustrate a decrease of the conductivity within the EG monolayer due to generation of hydroxylic and carboxylic groups in the graphene lattice. $C_{D L}$ (Fig. 1D) showed significantly larger values than $C_{I}$ (Fig. 2SB). The monitoring of the fitted values of the exponent factor for both CPEs (Fig. 2SD) showed values close to 1 for $\phi_{D L}$ at the anodization times corresponding to the maximum values of $C_{D L}$, which indicates a pure capacitance representation of the CPE in this time interval. These facts lead to the interpretation of $C_{D L}$ as the normal electric double layer capacitance on the anodized EG. At the end of the increase of $R_{S}$, at about 120s, there is a sudden increase of $C_{D L}$ (Fig. 1D). This might be related to fracturing of the EG layer and the formation of reactive defect sites [15]. At the same 
time, the increase of the double layer capacitance might be associated with the removal of contaminants on the EG surface.

$C_{D L}$ exhibited a peak-shaped time dependence during the anodization (Fig. 1D). The descending branch of the double layer capacitance probably illustrates a change of the surface morphology at long anodization times. Optimization of the anodization time opens up the possibility to maximize the double layer capacitance on the EG. The highest value of the double layer capacitance is about $22 \mu \mathrm{F}$, which was obtained at 140-175 s of anodization yielding an increase of the double layer capacitance by a factor of 21 compared to the pristine EG. This corresponds to an extreme value of the optimized specific capacitance: $701 \mu \mathrm{F} \mathrm{cm}{ }^{-2}$. This value is much higher than those obtained for other carbon materials utilized for electrical charge storage $[27,28]$, which indicates high availability of the material and efficient modification of the graphene with defects of high capacitance achieved via optimized anodization. Since the capacitance of graphene is directly proportional to the number of exposed edge planes [29, 30], it is possible to estimate the modification efficiency as 21, which is larger than the value of Loh's group [15] (about 7.5) achieved after $500 \mathrm{~s}$ of anodization.

There are several possible reasons for obtaining specific capacitance values significantly larger than those reported by Loh et al. using voltammetry. Firstly, here we report the value of the double layer capacitance obtained at an optimized anodization time. This optimized capacitance only appears in a limited time range (140-175 s). The shorter and longer times are characterized by smaller values of the double layer capacitance due to an incomplete modification and change of the surface morphology, respectively. Secondly, the EG studied here has been developed via thermal annealing in argon atmosphere, which leads to a monolayer-dominated formation of the graphene. The values of the specific capacitance are affected by the method of EG fabrication due to the different contributions of defects of higher DOS and higher capacitance. Thirdly, the pulsed anodization technique utilized in this study might provide a different anodization effect than in the previous studies. 
It should be noticed that the fabrication of graphene layers from bulk materials, such as graphene oxide and its derivatives, can decrease the specific capacitance in comparison with a graphene monolayer due to the contribution of the $\mathrm{sp}^{2}$-type basal plane of lower DOS and lower capacitance [29].

Our findings suggest that controlled anodization of EG may allow fabrication of a material with very high charge storage capacity. However, since the gravimetric specific capacitance is reversely proportional to the mass of the whole crystal, a decrease of the content of silicon carbide, which might e.g. be achieved via anodization of EG fabricated on nanoparticles, should be done on the way to applications.

\subsection{Voltammetric study of redox reactions on pristine and anodized EG}

The change of the electrochemical activity of the EG due to anodization has been studied with electrode reaction kinetics of reversible redox probes. Fig. 2A shows a series of backgroundsubtracted voltammetric responses of the $\mathrm{Fe}(\mathrm{CN}) 6^{3-/ 4-}$ redox couple, undergoing reversible single electron exchange at pristine EG as working electrode, obtained with different scan rates. It is seen that the redox process is characterized by sluggish quasi-reversible kinetics with peak-to-peak separation potentials between 0.6 and $0.8 \mathrm{~V}$. The absence of any significant redox signal midway between the separated redox peaks indicates a lack of contribution from defects since they would possess fast electrode kinetics [6, 31, 32]. A pure diffusion control of the redox process was confirmed by a scan rate study (Fig. 3S). The overall electron transfer rate constant was estimated by the Matsuda-Ayabe method [33,34] and was found to be in the range $2-7 \times 10^{-7} \mathrm{~cm} \mathrm{~s}^{-1}$ (Supporting Note 1).

A significant decrease of the peak-to-peak potential separation as well as a substantial peak current increase is observed for $\mathrm{Fe}(\mathrm{CN})_{6}^{3-4-}$ voltammetric response at the anodized EG (Fig. 2B), illustrating an activation of the electrode, which correlates with the impedance data. This is in agreement with previous results from Loh's group [15]. Thus, the anodization leads both to an 
increase of the double layer capacitance and to significant changes of the electrochemical activity of the surface.

Since the surface state of freshly-cleaved HOPG is affected by exposure to the environment [20], the change of the $\mathrm{Fe}(\mathrm{CN})_{6}^{3-/ 4-}$ redox process kinetics on EG caused by anodization might, in addition to the structural changes, be affected by the removal of contaminants from the surface. An inactivation of the surface was observed for continuous cycling of $\mathrm{Fe}(\mathrm{CN}) 6^{3-/ 4-}$ redox process on anodized EG as a continuous increase of the peak-to-peak potential separation, which is in agreement with observations for HOPG reported by Unwin’s group [20]. The electron transfer rate constant, estimated by a simulation of the background-subtracted voltammogram (see Fig. 2B), was $0.29 \mathrm{~cm} \mathrm{~s}^{-1}$, which is higher than the value of $0.01 \mathrm{~cm} \mathrm{~s}^{-1}$ obtained by Loh's group for anodized EG using the Nicholson method [15]. The difference might be due to the different methods of electrode kinetics assessment as well as due to the pulsed anodization procedure utilized here. The $\mathrm{Ru}\left(\mathrm{NH}_{3}\right)_{6}{ }^{3+/ 2+}$ redox couple was also used as a redox probe for the study of changes in the electrode reaction kinetics on EG due to the anodization. This probe is less sensitive to changes of the surface states than $\mathrm{Fe}(\mathrm{CN}) 6^{3-/ 4-}$ redox couple $[15,20]$. Fig. 2C represents a series of background-subtracted cyclic voltammetric responses obtained in the presence of the redox probe with different scan rates at pristine EG as the working electrode. It is seen that the $\mathrm{Ru}\left(\mathrm{NH}_{3}\right)_{6}{ }^{3+/ 2+}$ redox couple is characterized by a semi-reversible redox process with peak-to-peak separation potentials up to $0.2 \mathrm{~V}$. A pure diffusion control of the redox process was confirmed by a scan rate study (Fig. 5S). The slopes of plots of the $\log _{10}$ of the observed peak currents versus $\log _{10}$ of the scan rate (Fig. 6S) were 0.47 and 0.46 for anodic and cathodic peak currents, respectively, indicating semi-infinite diffusion. This can be modelled by the Randles-Sevcik equation [35] and allows applying the Nicholson method [36] for the assessment of the electron transfer rate constant (Supporting Note 2). The values of the electron transfer rate constant, $k^{0}$, for the pristine EG were in the range $0.7-1.2 \times 10^{-3} \mathrm{~cm} \mathrm{~s}^{-1}$, which is much larger than for the $\mathrm{Fe}(\mathrm{CN})_{6}{ }^{3-/ 4-}$ redox process. 
The anodization of the EG did not affect the kinetics of $\mathrm{Ru}\left(\mathrm{NH}_{3}\right)_{6}{ }^{3+/ 2+}$ redox process (Fig. 2D). Nor did the peak-to-peak potential separation change significantly with anodization and this resulted in small changes in the electron transfer rate constant. Table 1 summarizes the electrochemical parameters obtained in this study. This effect illustrates the insensitivity of $\mathrm{Ru}\left(\mathrm{NH}_{3}\right)_{6}{ }^{3+/ 2+}$ redox process to changes of the surface state. The results are in agreement with those of Loh's group, supporting their finding that electron transfer kinetics of $\mathrm{Fe}(\mathrm{CN}) 6^{3-/ 4-}$ redox process correlates directly with the defect density of epitaxial graphene, while those of $\mathrm{Ru}\left(\mathrm{NH}_{3}\right)_{6}{ }^{3+/ 2+}$ redox process do not.

\subsection{Characterization of pristine and anodized EG with reflectance mapping and Raman} spectroscopy

In order to investigate the anodization induced structural changes of the EG reflectance and Raman mapping were utilized. Reflectance maps displaying the graphene thickness [23] were recorded near the center of the sample before and after the anodization procedure (Fig. 3). The morphology in terms of graphene thickness is very similar in the two maps, as is the monolayer - bilayer distribution ( $\sim 80 \%-\sim 20 \%$, respectively), indicating that the mapped $30 \times 30 \mu \mathrm{m}^{2}$ random areas are representative for the morphology of the whole sample. Raman mapping (Fig. 4) comprising 256 spectra was performed on a smaller area (indicated by the squares on both maps (Fig. 3)) chosen so as to provide sufficient statistics on the measurements over both bilayer and monolayer regions. The smaller reflectance maps displayed under the larger maps in Fig. 3 are collected simultaneously with the Raman mapping and used for separating the spectra from monolayer from the spectra from bilayer regions. These smaller maps also confirm that the intended areas chosen from the larger maps are indeed sampled by Raman, i.e. no sample drift occurs during the measurements. The G, D and 2D peaks of each Raman spectrum were fitted with single Lorentzian line-shapes in order to estimate the corresponding peak positions, amplitudes, linewidths and the 
ratio of D- and G-peak intensities, $\mathrm{I}(\mathrm{D}) / \mathrm{I}(\mathrm{G})$, utilized for the assessment of the defect concentration in the monolayer region of the anodized sample.

The Raman spectra recorded before and after anodization of the EG sample (Fig. 4A) show very distinct differences in the monolayer and bilayer regions of the sample. The spectra from the pristine EG samples are typical for sublimation-grown graphene on $\mathrm{SiC}$ and display, apart from the dominating $\mathrm{G}$ and $2 \mathrm{D}$ peaks, also a contribution from the buffer layer (BL) underlying the graphene. The typical linewidth, full width at half maximum (FWHM) of the 2D peak in the monolayer region of the pristine EG is in the range of $31-35 \mathrm{~cm}^{-1}$, and the FWHM of the G peak is about $30 \mathrm{~cm}^{-1}$. The D-peak associated with the presence of defects in graphene can hardly be discerned in the spectra of the pristine sample for both mono- and bilayer regions. The D-peak overlaps with the contribution from the BL in the Raman spectra. However, the monolayer region after the anodization exhibits severe changes. The strong D peak, as well as the appearance of the $\mathrm{D}^{\prime}$ peak and the combined $\mathrm{D}+\mathrm{D}^{\prime}$ band in the spectra, are evidence of a significant increase in the defect density as a result of the anodization. The 2D peak in the monolayer region also broadens to more than $40 \mathrm{~cm}^{-1}$ (from $31-35 \mathrm{~cm}^{-1}$ in the pristine sample). Interestingly, no detrimental changes in the Raman spectrum can be detected in the bilayer region of the anodized sample. The D peak remains undetectable as in the case of the pristine EG, albeit some changes in the structure of the BL part of the spectrum can be noticed.

Fig. 4B displays the $2 \mathrm{D}$ versus $\mathrm{G}$ peak positions obtained on the different points of the monolayer and the bilayer regions on pristine and anodized samples. The 2D vs. G peak positions of the pristine sample are scattered around a line parallel to the pure strain line representing the variation of 2D vs. G-peak positions for suspended graphene [37] (the solid straight line of Fig. 4B, corrected for the $532 \mathrm{~nm}$ excitation used in our work). The slopes of both lines are essentially determined by the ratio of the Grüneisen parameters of the $2 \mathrm{D}$ and $\mathrm{G}$ peaks. The fact that they are parallel suggests that the scatter of the points in the pristine EG is mainly due to variations in strain, while 
the doping level can be assumed constant. However, the observed linear dependence of $2 \mathrm{D}$ vs. G positions for pristine EG appears shifted with respect to the data obtained for suspended graphene. This circumstance is not understood at present and hinders separate determination of strain and doping conditions along the lines [37]. Nevertheless, the electron doping in the pristine sample can be assumed to be in the range $2 \times 10^{12}-1 \times 10^{13} \mathrm{~cm}^{-2}$, typically measured in similar samples using angular-resolved photoelectron spectroscopy (ARPES)[38]. The 2D-peak position is virtually the same in this range of n-type doping [39]. Therefore, the large downshift of the 2D peak in the anodized sample $\left(20-40 \mathrm{~cm}^{-1}\right)$ indicates a large increase in the electron concentration. This increase should be accompanied by some upshift in the G-values of the order of $10 \mathrm{~cm}^{-1}$ [39]. However, the G-peak upshift saturates with the electron concentration increase and may be compensated by relief in the strain compared to the pristine sample due to the creation of edge defects breaking the graphene network during the anodization. The 2D peak also downshifts with decreasing strain. However, the strong decrease of the slope of the 2D vs. G peak position line of the monolayer after anodization (the dotted line of Fig. 4B) suggests that most of the 2D-peak downshift is due to increased doping and its variation. Assuming a downshift in 2D peak position of the order of $20-40 \mathrm{~cm}^{-1}$, a rough estimation of the doping variation [39] gives the range $3.5-$ $4 \times 10^{13} \mathrm{~cm}^{-2}$ in the anodized sample. This drastic increase in doping is due to the presence of edge defects and is the main reason for the observed increase in the capacitance of the anodized sample.

The I(D)/I(G) ratio for the anodized monolayer varies between 0.8 and 1.3 approximately, which corresponds to crystallite sizes (denoted hereafter as $L_{d}$ ) between 10 and $13 \mathrm{~nm}$ [40]. By crystallite size, we mean the average distance between two subsequent disruptions in the graphene network forming edge defects and activating the $\mathrm{D}$ peak. The graphene between these two defects is the intact crystallite. The estimated crystallite sizes allow a rough estimation of the corresponding electron concentrations due to the presence of dangling bonds at the edges of the disrupted graphene lattice. This can be done by assuming that each dangling bond contributes one electron to the doping concentration, and the number of dangling bonds is made (i.e. lattice sites) along the 
two perpendicular sides of a square of size $L_{d}$ as a primitive model yielding the correct order of magnitude for the edge defect concentration. The two perpendicular sides of the square along which the counting of dangling bonds (i.e., lattice sites) is made can generally be considered as oriented along the "zig-zag” and the "armchair" directions of the graphene lattice, which have somewhat different densities of lattice sites. Thus, if $a_{\mathrm{z}}=0.246 \mathrm{~nm}$ denotes the in-plane lattice constant of graphene, the lattice period in the zig-zag direction is simply $a_{\mathrm{z}}$, whereas that in the armchair direction is $2 a_{z} / \sqrt{3}$. Thus, for the purpose of estimation of the number of dangling bonds in the disrupted graphene, we replace the lattice with a regular set of squares forming a quasilattice with period $L_{d}$ in both armchair and zig-zag directions. It should be noted that only one armchair side and one zig-zag side per square will contribute to the electron concentration, because this is the motif of the square "unit cell" in our model. Consequently, the number of dangling bonds (electrons) per square divided by the area of the square $L_{d}^{2}$ gives the electron concentration $n_{e}$ :

$$
n_{e}=\left(\frac{L_{d}}{a_{z}}+2 \frac{L_{d} \sqrt{3}}{2 a_{z}}\right) \frac{1}{L_{d}^{2}}
$$

Here, the factor 2 in front of the second term in parentheses accounts for the two lattice sites per period in the armchair direction. The concentrations calculated from Eq. (2) are $n_{e} \approx 8 \times 10^{13} \mathrm{~cm}^{-2}$ for $L_{d}=10.5 \mathrm{~nm}$, and $n_{e} \approx 6.5 \times 10^{13} \mathrm{~cm}^{-2}$ for $L_{d}=13.4 \mathrm{~nm}$, respectively. The obtained estimates are in very good agreement with the expectation $n_{e} \approx 4 \times 10^{13} \mathrm{~cm}^{-2}$ based solely on the observed large downshift of the 2D peak. This agreement corroborates our notion that the increased doping is due to creation of edge defects upon anodization, which contributes to the increased specific capacitance of graphene.

Surprisingly, the Raman spectra from the bilayer region show no discernible contribution from the D peak, suggesting that the formation of defects is limited to the monolayer graphene. In addition, 
a systematic study of the doping dependence of the Raman peaks in bilayer graphene is not available in the literature, to the best of our knowledge. For this reason, and since the bilayer contribution in our sample is limited to $\sim 20 \%$, here we only analyze the results obtained from the monolayer.

The AFM measurements allowed the qualitative analysis of morphology change of EG due to the anodization (Fig. 7S). The smooth surface morphology was observed for pristine EG, while the anodization led to the appearance of visible nanoscale defects uniformly distributed over the surface of study.

\section{Conclusions}

Creation of reactive defects on an epitaxial graphene monolayer on silicon carbide was achieved via anodization. In addition a surface cleaning likely occurs during the anodization. Electrochemical monitoring of the double layer capacitance during anodization allows optimization of the activation process. The combination of extreme smoothness, robustness and efficient surface cleaning and modification by reactive defects gives a significant increase of the reactivity and an up to 21 fold increase in charge storage capacity of graphene. Contrary to the $\mathrm{Ru}\left(\mathrm{NH}_{3}\right)_{6}{ }^{3+/ 2+}$ redox process, $\mathrm{Fe}(\mathrm{CN})_{6}{ }^{3-/ 4-}$ redox couple revealed irreversible changes of the kinetics accompanied with a dramatic increase of the electrode reaction rate due to the anodization. Raman spectroscopy showed that the defect formation occurred only in the graphene monolayer, which represents $80 \%$ of the surface. The bilayer areas do not seem to contribute with any anodization induced defect formation. The defect density is estimated to be of the order of $4-8 \times 10^{13}$ $\mathrm{cm}^{-2}$, which roughly corresponds to $2 \%$ of the carbon atoms in the monolayer area. 


\section{References}

[1] A. Ambrosi, M. Pumera, The Structural Stability of Graphene Anticorrosion Coating Materials is Compromised at Low Potentials, Chemistry-a European Journal, 21 (2015) 7896-7901.

[2] M. Pumera, Electrochemistry of Graphene: New Horizons for Sensing and Energy Storage, Chemical Record, 9 (2009) 211-223.

[3] Y.Y. Shao, J. Wang, H. Wu, J. Liu, I.A. Aksay, Y.H. Lin, Graphene Based Electrochemical Sensors and Biosensors: A Review, Electroanalysis, 22 (2010) 1027-1036.

[4] I.P.O. UK, Graphene. The worldwide patent landscape in 2013, Intellectual Property Office UK, 2013.

[5] Y. Wang, Z. Shi, Y. Huang, Y. Ma, C. Wang, M. Chen, Y. Chen, Supercapacitor Devices Based on Graphene Materials, Journal of Physical Chemistry C, 113 (2009) 13103-13107.

[6] D.A.C. Brownson, D.K. Kampouris, C.E. Banks, Graphene electrochemistry: fundamental concepts through to prominent applications, Chemical Society Reviews, 41 (2012) 6944-6976.

[7] R.L. McCreery, Advanced carbon electrode materials for molecular electrochemistry, Chemical Reviews, 108 (2008) 2646-2687.

[8] N.G. Shang, P. Papakonstantinou, M. McMullan, M. Chu, A. Stamboulis, A. Potenza, S.S. Dhesi, H. Marchetto, Catalyst-Free Efficient Growth, Orientation and Biosensing Properties of Multilayer Graphene Nanoflake Films with Sharp Edge Planes, Advanced Functional Materials, 18 (2008) 3506-3514.

[9] C. Tan, J. Rodrıguez-Lopez, J.J. Parks, N.L. Ritzert, D.C. Ralph, H.D. Abruna, Reactivity of Monolayer Chemical Vapor Deposited Graphene Imperfections Studied Using Scanning Electrochemical Microscopy, ACS Nano, 6 (2012) 3070-3079.

[10] A. Ambrosi, A. Bonanni, Z. Sofer, M. Pumera, Large-scale quantification of CVD graphene surface coverage, Nanoscale, 5 (2013) 2379-2387.

[11] X. Huang, Z. Zeng, Z. Fan, J. Liu, H. Zhang, Graphene-Based Electrodes, Advanced Materials, 24 (2012) 5979-6004. 
[12] A. Reina, X.T. Jia, J. Ho, D. Nezich, H.B. Son, V. Bulovic, M.S. Dresselhaus, J. Kong, Large Area, Few-Layer Graphene Films on Arbitrary Substrates by Chemical Vapor Deposition, Nano Letters, 9 (2009) 30-35.

[13] E. Dubuisson, Z. Yang, K.P. Loh, Optimizing Label-Free DNA Electrical Detection on Graphene Platform, Analytical Chemistry, 83 (2011) 2452-2460.

[14] K.V. Emtsev, A. Bostwick, K. Horn, J. Jobst, G.L. Kellogg, L. Ley, J.L. McChesney, T. Ohta, S.A. Reshanov, J. Roehrl, E. Rotenberg, A.K. Schmid, D. Waldmann, H.B. Weber, T. Seyller, Towards wafer-size graphene layers by atmospheric pressure graphitization of silicon carbide, Nature Materials, 8 (2009) 203-207.

[15] C.X. Lim, H.Y. Hoh, P.K. Ang, K.P. Loh, Direct Voltammetric Detection of DNA and pH Sensing on Epitaxial Graphene: An Insight into the Role of Oxygenated Defects, Analytical Chemistry, 82 (2010) 7387-7393.

[16] R. Yakimova, C. Virojanadara, D. Gogova, M. Syvajarvi, D. Siche, K. Larsson, L.I. Johansson, Analysis of the Formation Conditions for Large Area Epitaxial Graphene on SiC Substrates, in: A.J. Bauer, P. Friedrichs, M. Krieger, G. Pensl, R. Rupp, T. Seyller (Eds.) Silicon Carbide and Related Materials 2009, Pts 1 and 22010, pp. 565-568.

[17] A. Tzalenchuk, S. Lara-Avila, A. Kalaboukhov, S. Paolillo, M. Syvajarvi, R. Yakimova, O. Kazakova, T.J.B.M. Janssen, V. Fal'ko, S. Kubatkin, Towards a quantum resistance standard based on epitaxial graphene, Nature Nanotechnology, 5 (2010) 186-189.

[18] J.G.S. Moo, A. Ambrosi, A. Bonanni, M. Pumera, Inherent Electrochemistry and Activation of Chemically Modified Graphenes for Electrochemical Applications, Chemistry-an Asian Journal, 7 (2012) 759-770.

[19] C.Y. Lee, A.M. Bond, Evaluation of Levels of Defect Sites Present in Highly Ordered Pyrolytic Graphite Electrodes Using Capacitive and Faradaic Current Components Derived Simultaneously from Large-Amplitude Fourier Transformed ac Voltammetric Experiments, Analytical Chemistry, 81 (2009) 584-594. 
[20] A.N. Patel, M.G. Collignon, M.A. O'Connell, W.O.Y. Hung, K. McKelvey, J.V. Macpherson, P.R. Unwin, A New View of Electrochemistry at Highly Oriented Pyrolytic Graphite, Journal of the American Chemical Society, 134 (2012) 20117-20130.

[21] T. Burnett, R. Yakimova, O. Kazakova, Mapping of Local Electrical Properties in Epitaxial Graphene Using Electrostatic Force Microscopy, Nano Letters, 11 (2011) 2324-2328.

[22] R.C. Engstrom, V.A. Strasser, Characterization of Electrochemically Pretreated GlassyCarbon Electrodes, Analytical Chemistry, 56 (1984) 136-141.

[23] I.G. Ivanov, J.U. Hassan, T. Iakimov, A.A. Zakharov, R. Yakimova, E. Janzen, Layer-number determination in graphene on SiC by reflectance mapping, Carbon, 77 (2014) 492-500.

[24] M. O'Donoghue, R. Garrett, V. Datta, P. Roberts, T. Aben, Electrochemical impedance spectroscopy: Testing coatings for rapid immersion service, Materials Performance, 42 (2003) 3641.

[25] D. Loveday, P. Peterson, B. Rodgers, Evaluation of organic coatings with electrochemical impedance spectroscopy - Part 3: Protocols for testing coatings with EIS, Jct Coatingstech, 2 (2005) 22-27.

[26] V.D. Jovic, B.M. Jovic, EIS and differential capacitance measurements onto single crystal faces in different solutions - Part I: Ag(111) in $0.01 \mathrm{M} \mathrm{NaCl}$, Journal of Electroanalytical Chemistry, 541 (2003) 1-11.

[27] L.L. Zhang, R. Zhou, X.S. Zhao, Graphene-based materials as supercapacitor electrodes, Journal of Materials Chemistry, 20 (2010) 5983-5992.

[28] J. Zhu, D. Yang, Z. Yin, Q. Yan, H. Zhang, Graphene and Graphene-Based Materials for Energy Storage Applications, Small, 10 (2014) 3480-3498.

[29] M.S. Goh, M. Pumera, Multilayer graphene nanoribbons exhibit larger capacitance than their few-layer and single-layer graphene counterparts, Electrochemistry Communications, 12 (2010) 1375-1377. 
[30] T. Kim, S. Lim, K. Kwon, S.H. Hong, W.M. Qiao, C.K. Rhee, S.H. Yoon, I. Mochida, Electrochemical capacitances of well-defined carbon surfaces, Langmuir, 22 (2006) 9086-9088.

[31] D.A.C. Brownson, C.E. Banks, The electrochemistry of CVD graphene: progress and prospects, Physical Chemistry Chemical Physics, 14 (2012) 8264-8281.

[32] T.J. Davies, R.R. Moore, C.E. Banks, R.G. Compton, The cyclic voltammetric response of electrochemically heterogeneous surfaces, Journal of Electroanalytical Chemistry, 574 (2004) 123-152.

[33] A.J. Bard, L.R. Faulkner, Electrochemical Methods. Fundamentals and Applications, John Wiley and Sons, Inc, New York, 2001.

[34] H. Matsuda, Y. Ayabe, Z. Electrochem., 59 (1955) 494.

[35] R.G. Compton, C.E. Banks, Understanding Voltammetry, World Scientific Publishing Company2007.

[36] R.S. Nicholson, Theory and Application of Cyclic Voltammetry for Measurement of Electrode Reaction Kinetics, Analytical Chemistry, 37 (1965) 1351-1355.

[37] J.E. Lee, G. Ahn, J. Shim, Y.S. Lee, S. Ryu, Optical separation of mechanical strain from charge doping in graphene, Nature Communications, 3 (2012).

[38] D. Usachov, O. Vilkov, A. Gruneis, D. Haberer, A. Fedorov, V.K. Adamchuk, A.B. Preobrajenski, P. Dudin, A. Barinov, M. Oehzelt, C. Laubschat, D.V. Vyalikh, Nitrogen-Doped Graphene: Efficient Growth, Structure, and Electronic Properties, Nano Letters, 11 (2011) 54015407.

[39] A. Das, S. Pisana, B. Chakraborty, S. Piscanec, S.K. Saha, U.V. Waghmare, K.S. Novoselov, H.R. Krishnamurthy, A.K. Geim, A.C. Ferrari, A.K. Sood, Monitoring dopants by Raman scattering in an electrochemically top-gated graphene transistor, Nature Nanotechnology, 3 (2008) 210-215. 
[40] L.G. Cancado, A. Jorio, E.H. Martins Ferreira, F. Stavale, C.A. Achete, R.B. Capaz, M.V.O. Moutinho, A. Lombardo, T.S. Kulmala, A.C. Ferrari, Quantifying Defects in Graphene via Raman Spectroscopy at Different Excitation Energies, Nano Letters, 11 (2011) 3190-3196. 
Table 1. The electrochemical parameters estimated for pristine and anodized EG.

\begin{tabular}{|c|c|c|c|}
\hline \multirow{2}{*}{ Parameter } & & \multicolumn{2}{|c|}{ EG } \\
\hline & & Pristine & Anodized \\
\hline \multicolumn{2}{|l|}{ Capacitance, $\mu \mathrm{F} \mathrm{cm}{ }^{-2}$} & 33 & $7.0 \times 10^{2}$ \\
\hline \multirow{2}{*}{$\begin{array}{l}\text { Electron transfer rate } \\
\text { constant of } \mathrm{Fe}(\mathrm{CN})_{6}{ }^{3-/ 4-} \\
\text { redox process, } \mathrm{cm} \mathrm{s}^{-1}\end{array}$} & Matsuda-Ayabe method & $2-7 \times 10^{-7}$ & - \\
\hline & Simulation & - & 0.29 \\
\hline $\begin{array}{l}\text { Electron transfer rate } \\
\text { constant of } \\
\mathrm{Ru}\left(\mathrm{NH}_{3}\right)_{6}{ }^{3+/ 2+} \text { redox } \\
\text { process, } \mathrm{cm} \mathrm{s}^{-1}\end{array}$ & Nicholson method & $0.7-1.2 \times 10^{-3}$ & $0.8-1.4 \times 10^{-3}$ \\
\hline
\end{tabular}




\section{Figure legends}

Figure 1. Monitoring of EG anodization. A: impedance spectra in complex capacitance coordinates obtained for pristine ( $\square$ ) and anodized EG (O - $15 \mathrm{~s}, \triangle-30 \mathrm{~s} ; 0.1 \mathrm{M} \mathrm{KNO}_{3}, 0 \mathrm{~V}, 50$ $\mathrm{kHz}-1 \mathrm{~Hz}, 5 \mathrm{mV}$ amplitude). B: examples of impedance spectra obtained at anodized EG electrodes (45 s, 105 s, 165 s, 275 s and 490 s of anodization) in Nyquist coordinates; solid lines represent fitted spectra; Inset: equivalent circuit. $\mathbf{C}$ and D: the anodization time dependencies of the fitted solution resistance and the double layer capacitance on anodized EG, respectively.

Figure 2. Electrochemical kinetics of mono-electronic redox processes on pristine and anodized EG. Background-subtracted voltammetric responses of the $\mathrm{Fe}(\mathrm{CN})_{6}{ }^{3-/ 4-}(\mathbf{A})$ and $\mathrm{Ru}\left(\mathrm{NH}_{3}\right)_{6}{ }^{3+/ 2+}(\mathbf{C})$ redox processes obtained at pristine EG (scan rates of $5 \mathrm{mV} / \mathrm{s}$ (black), $10 \mathrm{mV} / \mathrm{s}$ (red), $20 \mathrm{mV} / \mathrm{s}$ (blue), $50 \mathrm{mV} / \mathrm{s}$ (green), $80 \mathrm{mV} / \mathrm{s}$ (magenta) and $100 \mathrm{mV} / \mathrm{s}$ (dark yellow; $0.1 \mathrm{M} \mathrm{KCl,} 1 \mathrm{mM}$ redox probe). Cyclic voltammograms obtained at pristine (black curves) and EG anodized for $45 \mathrm{~s}$ and $310 \mathrm{~s}$ (red and blue curves, respectively) in the presence of $\mathrm{K}_{4} \mathrm{Fe}(\mathrm{CN})_{6}(\mathbf{B}, 50 \mathrm{mV} / \mathrm{s}$; dashed blue curve - simulated cyclic voltammogram) or $\mathrm{Ru}\left(\mathrm{NH}_{3}\right)_{6} \mathrm{Cl}_{3}(\mathbf{D}, 20 \mathrm{mV} / \mathrm{s}) ; 0.1 \mathrm{M} \mathrm{KCl}, 1 \mathrm{mM}$ redox probe.

Figure 3. Reflectance maps on random areas $\left(30 \times 30 \mu \mathrm{m}^{2}\right)$ near the center of the pristine (A) and anodized (B) EG. The monolayer (1LG) and bilayer (2LG) regions appear in different color, as denoted in the map on the left. The white spot in the map of the pristine sample is due to a micropipe in the substrate, while the white/dark spots in the anodized sample most probably originate from electrolyte residues. The smaller reflectance maps $\left(4.5 \times 4.5 \mu \mathrm{m}^{2}\right)$ obtained together with the Raman mapping are shown below the corresponding larger maps (pointed by arrows). The color-coded map of the I(D)/I(G) ratio for the anodized sample is also displayed. 
Figure 4. A: Typical Raman spectra obtained on the monolayer and bilayer regions of the pristine and anodized EG. BL denotes the contribution from the buffer layer observable in all spectra; B: graphs of the 2D- versus G-peak positions obtained from Raman mapping before and after anodization. The solid and dotted straight lines are discussed in the main text. 

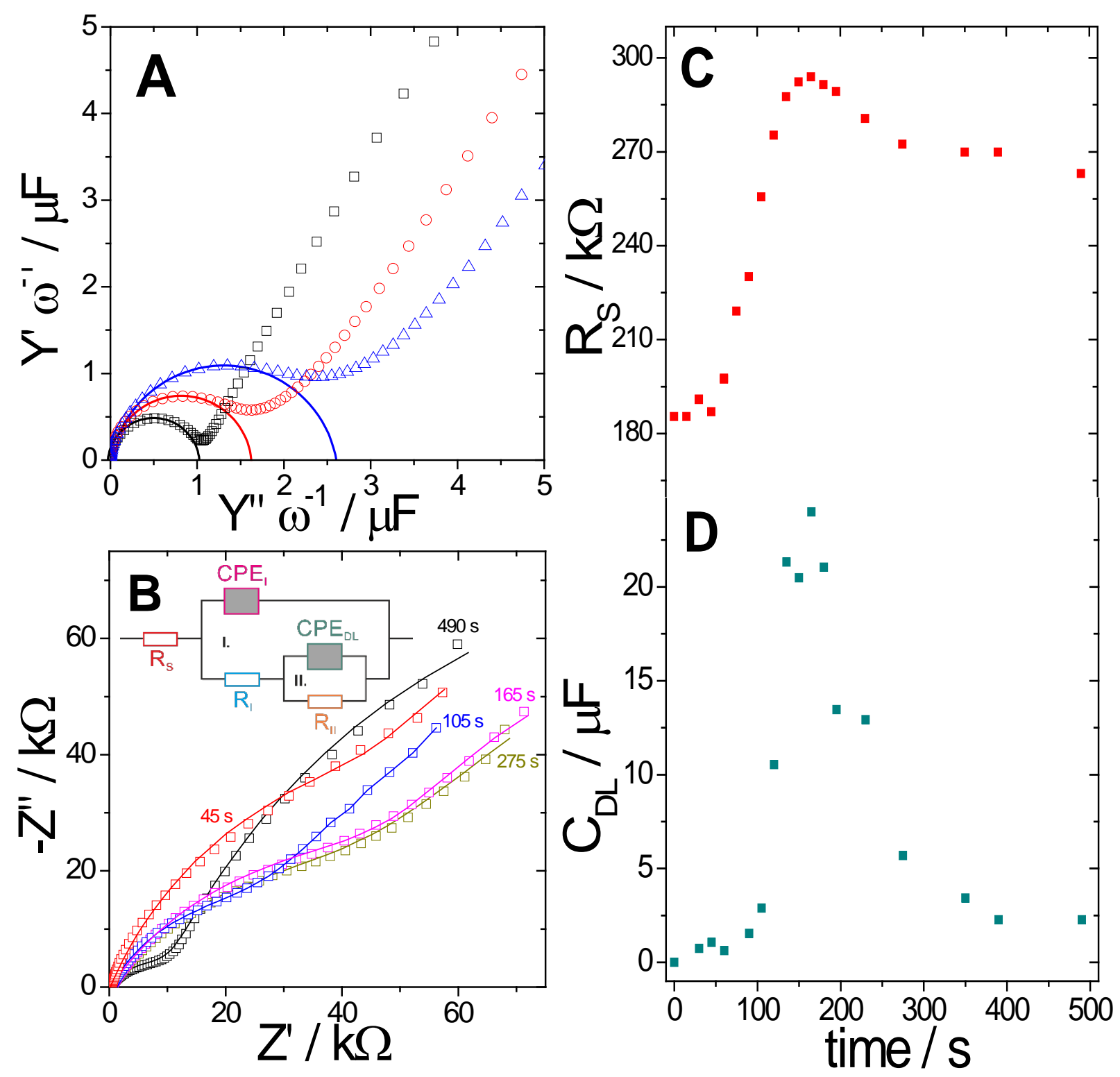

Figure 1 


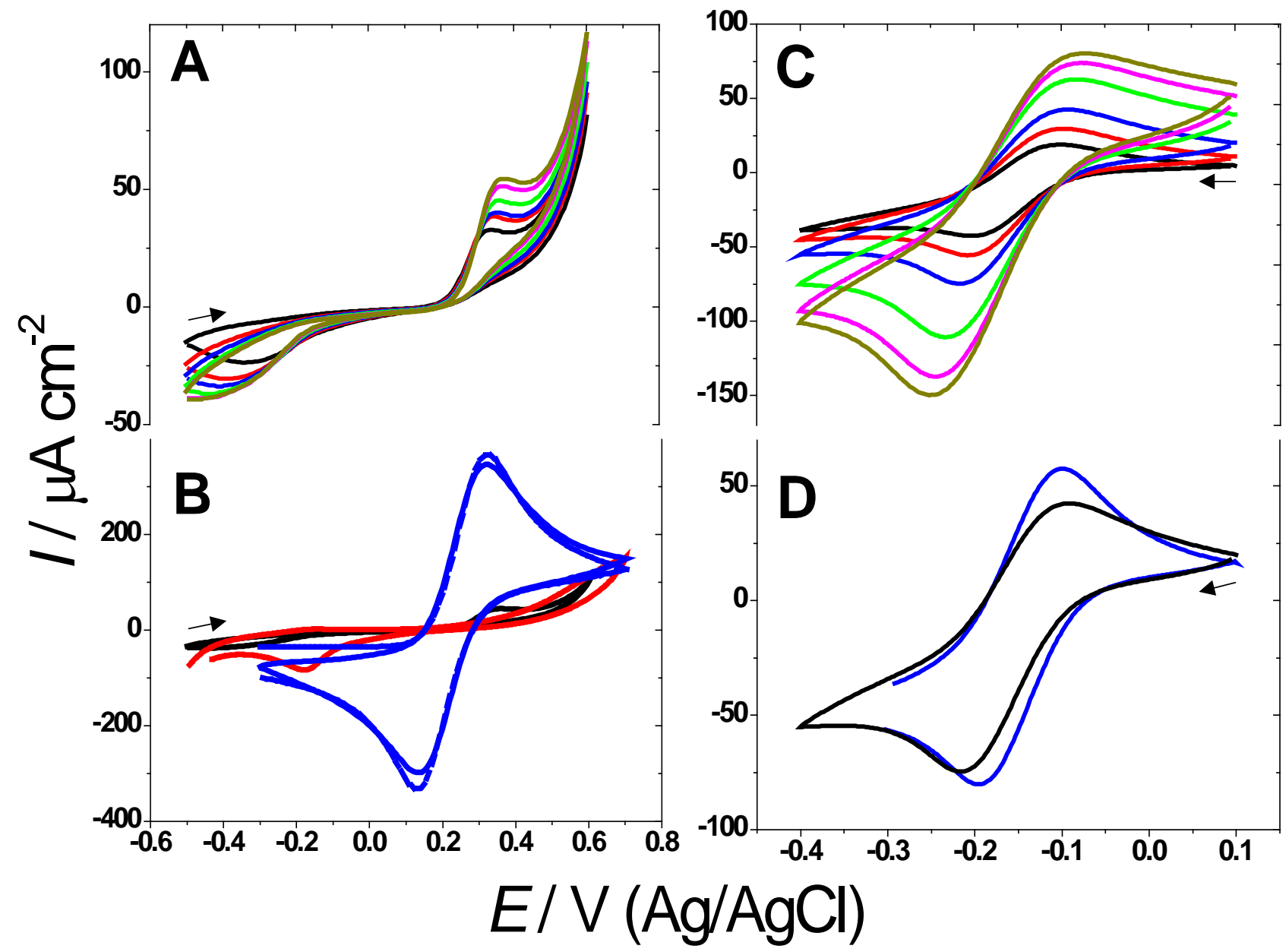

Figure 2 


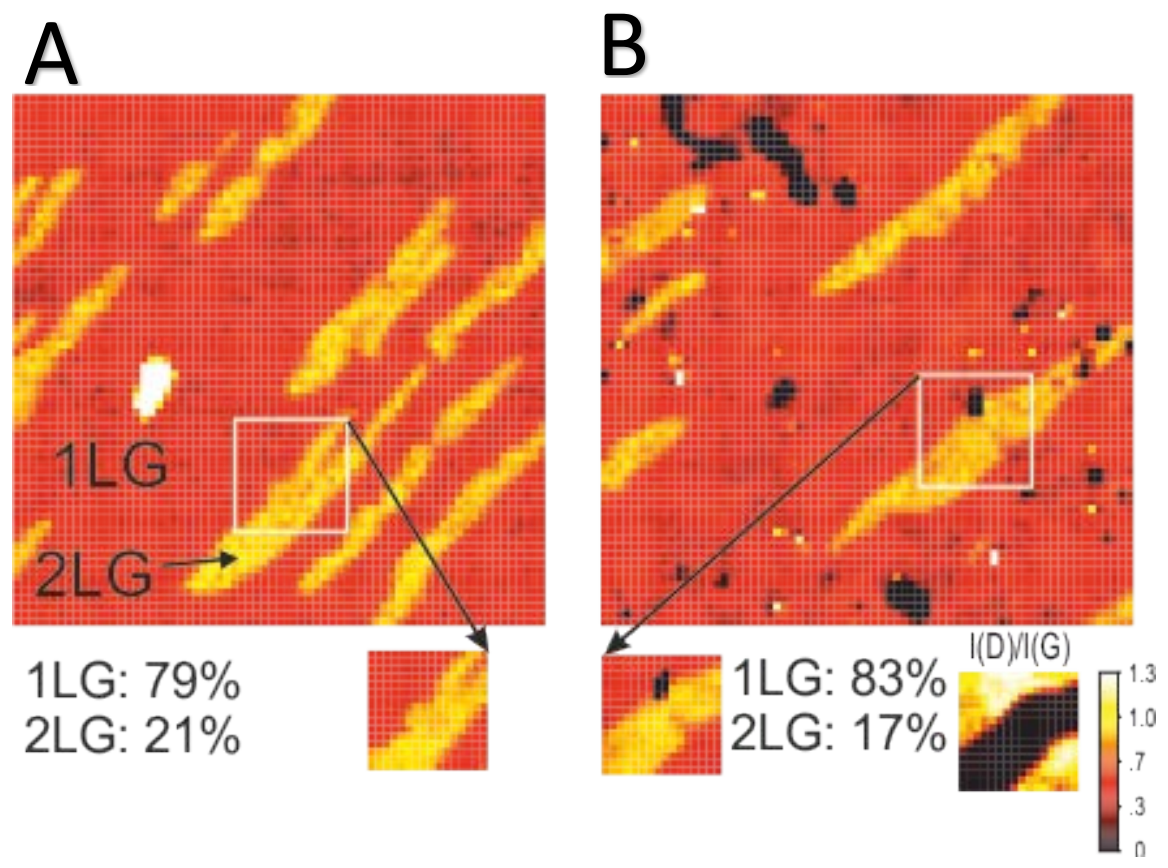

Figure 3 


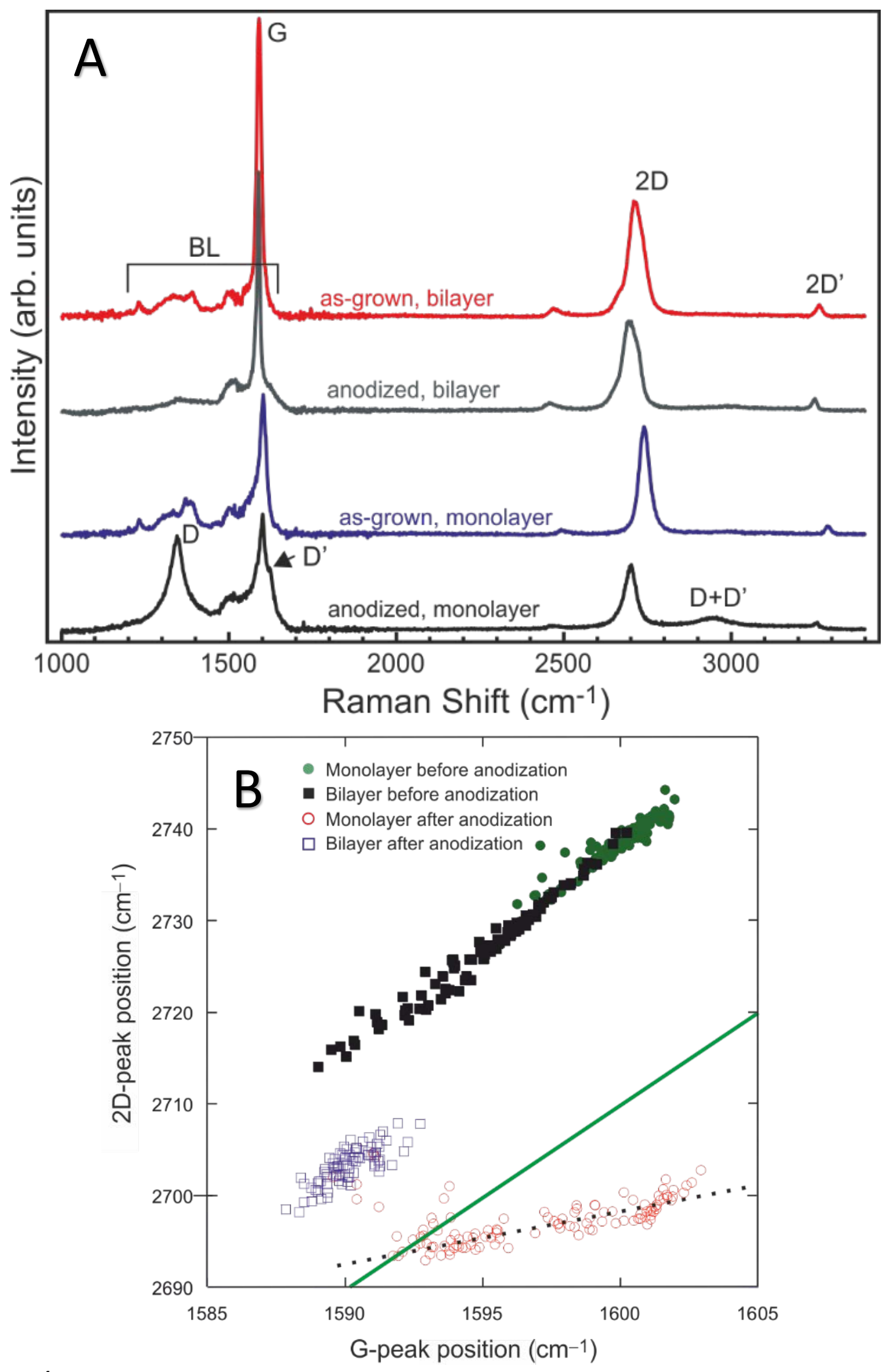

Figure 4 


\title{
SUPPORTING INFORMATION \\ Monitoring of epitaxial graphene anodization
}

\author{
Mikhail Yu. Vagin ${ }^{1,2 *}$, Alina N. Sekretaryova ${ }^{1,3}$, Ivan G. Ivanov ${ }^{1}$, Anna Håkansson ${ }^{1,2}$, Tihomir \\ Iakimov $^{1,4}$, Mikael Syväjärvi ${ }^{1,4}$, Rositsa Yakimova ${ }^{1,4}$, Ingemar Lundström ${ }^{1}$, Mats Eriksson ${ }^{1}$ \\ ${ }^{1}$ Department of Physics, Chemistry and Biology, Linköping University, SE-581 83, Linköping, \\ Sweden; e-mail: mikva@ifm.liu.se \\ ${ }^{2}$ Laboratory of Organic Electronics, Department of Science and Technology, Linköping \\ University, SE-601 74, Norrköping, Sweden \\ ${ }^{3}$ Department of Chemistry, Stanford University, Stanford, CA 94305-5080, USA \\ ${ }^{4}$ Graphensic AB, Teknikringen 1F, SE-58330 Linköping, Sweden.
}




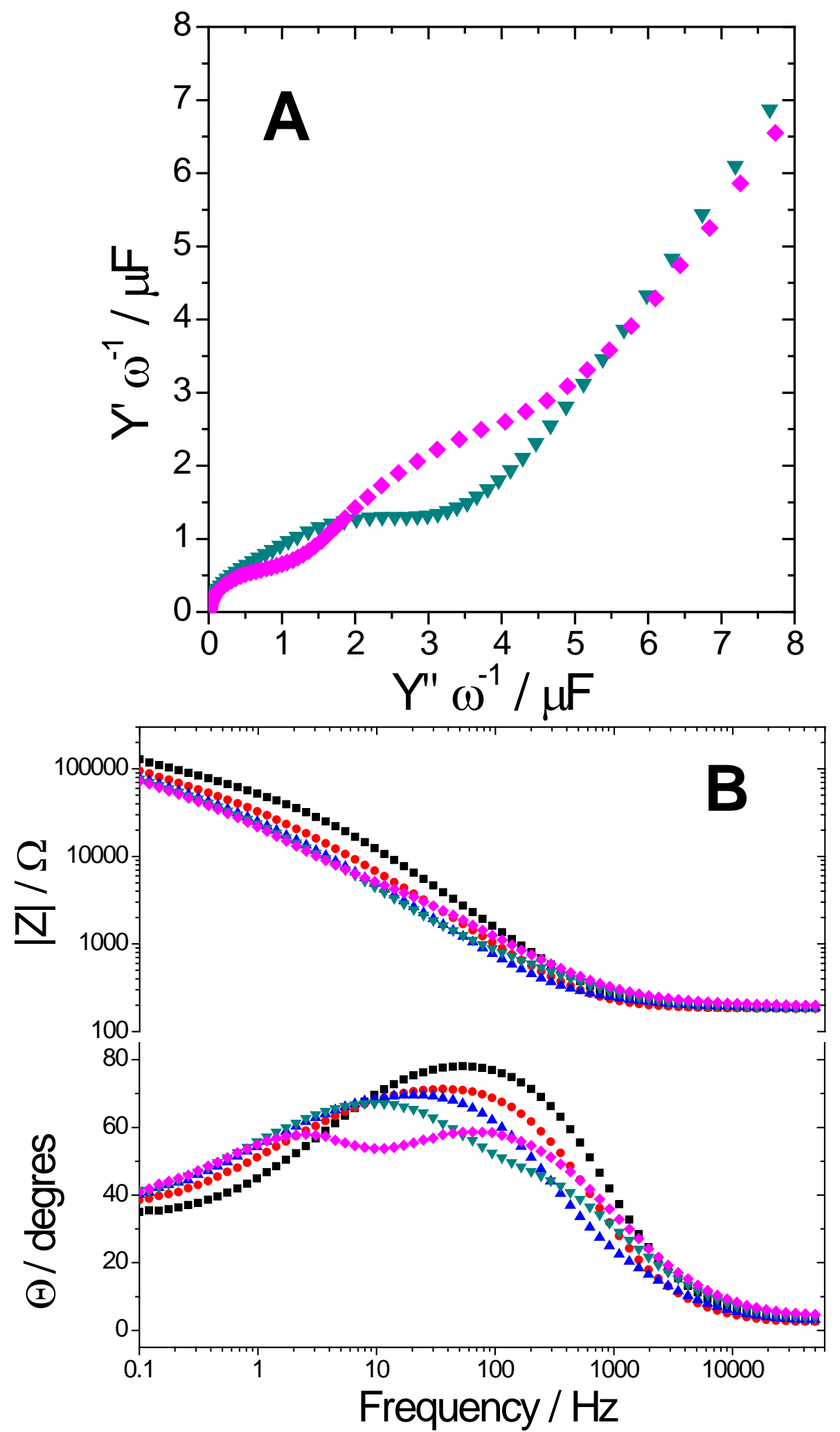

Figure 1S. Impedance spectra of anodized EG electrodes. A: spectra obtained after $45 \mathrm{~s}(\boldsymbol{\nabla})$ and $60 \mathrm{~s}(\diamond)$ of anodization in complex capacitance coordinates; B: Bode plot of impedance data obtained for pristine (অ) and anodized EG ( $-15 \mathrm{~s}, \boldsymbol{\Delta}-30 \mathrm{~s}, \boldsymbol{\nabla}-45 \mathrm{~s}, \bullet-60 \mathrm{~s} ; 0.1 \mathrm{M} \mathrm{KNO}_{3}$, $0 \mathrm{~V}, 50 \mathrm{kHz}-1 \mathrm{~Hz}, 5 \mathrm{mV}$ amplitude). 


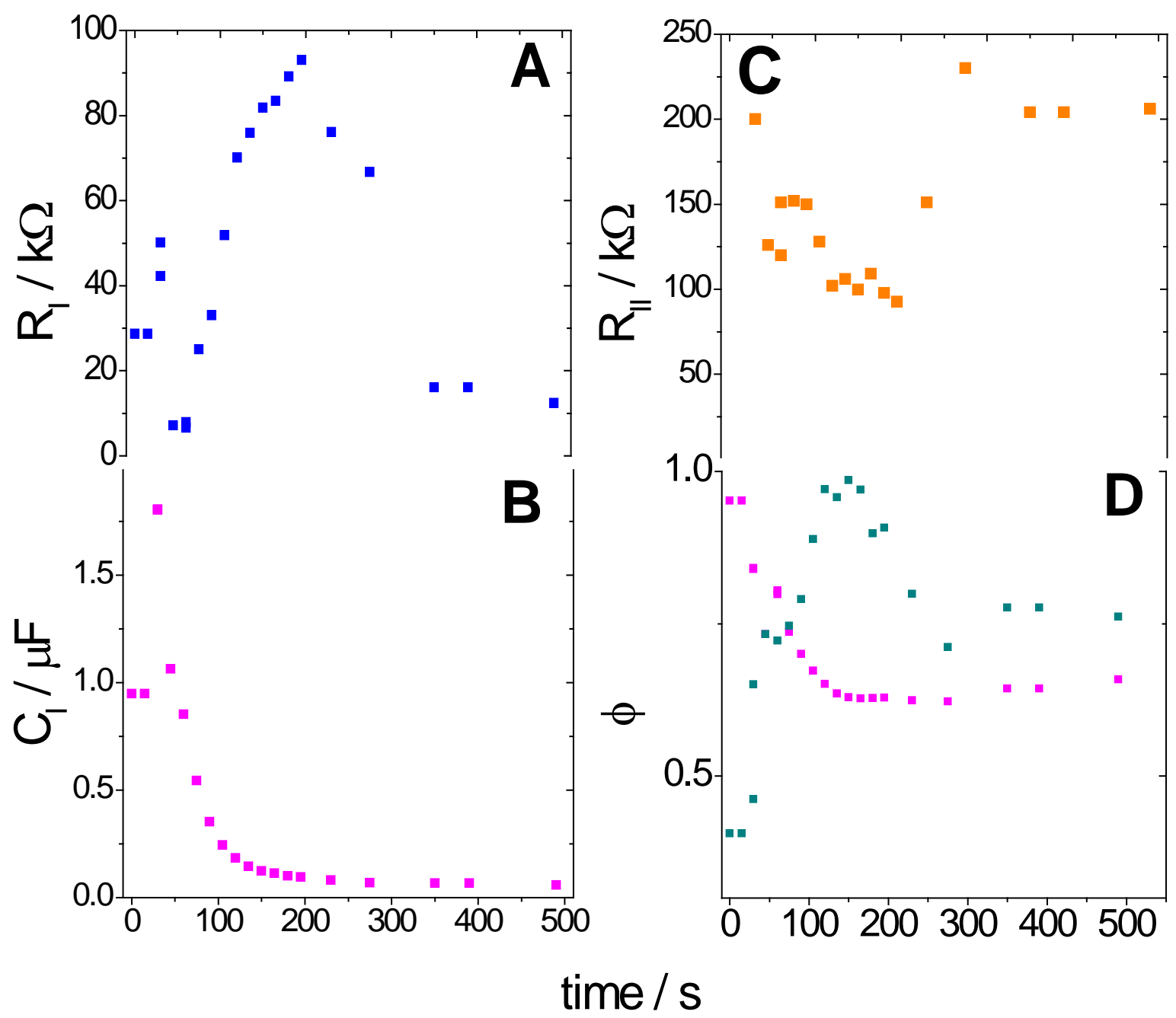

Figure 2S. The dependencies of fitted parameters on anodization time. $\mathrm{R}_{\mathrm{I}}(\mathbf{A})$ and $\mathrm{C}_{\mathrm{I}}(\mathbf{B})$ of the first and $\mathrm{R}_{\mathrm{II}}(\mathbf{C})$ for the second R-CPE unit of the equivalent circuit (see Fig. 1B); $\mathbf{D}$ : $\phi$ for $\mathrm{CPE}_{\mathrm{I}}$ $(\square)$ and $\mathrm{CPE}_{\mathrm{DL}}(\square)$. 

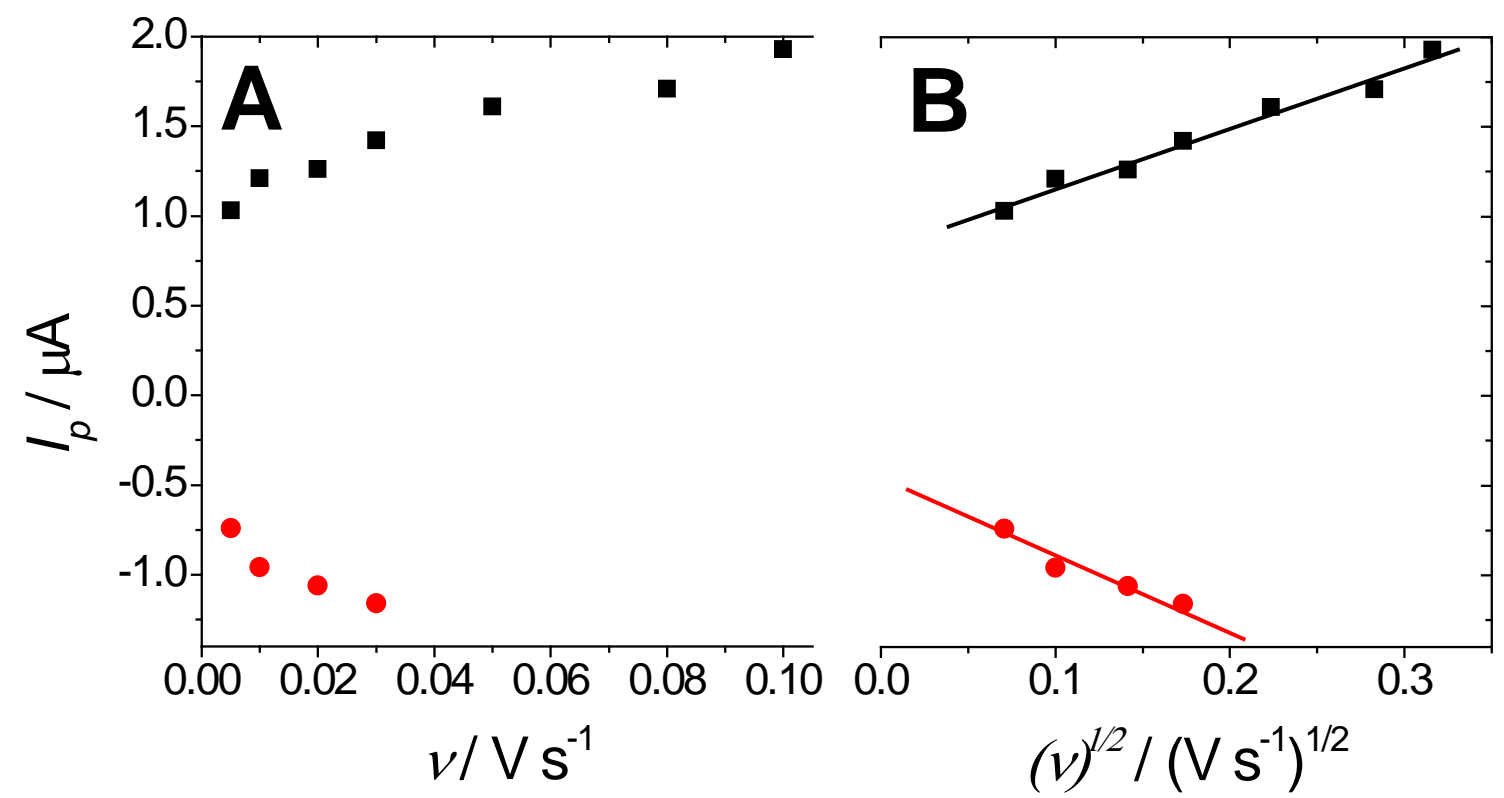

Figure 3S. Diffusion control of the ferro/ferricyanide redox process on pristine EG. The dependencies of the anodic $(\mathbf{\square})$ and cathodic $(\bullet)$ peak currents on the scan rate $(\mathbf{A})$ and on the square root of the scan rate $(\mathbf{B})\left(0.1 \mathrm{M} \mathrm{KCl}, 1 \mathrm{mM} \mathrm{K}{ }_{4} \mathrm{Fe}(\mathrm{CN})_{6}\right)$. Linear dependence of anodic and cathodic peak currents on the square root of scan rate demonstrates a pure diffusion control of the redox process. 


\section{Supporting Note 1. Electron transfer rate estimation by the Matsuda-Ayabe method.}

The overall electron transfer rate constant was estimated by the Matsuda-Ayabe method [1, 2]. The dimensionless parameter $\Xi(\Lambda, \alpha)$ was calculated as:

$$
\Xi(\Lambda, \alpha)=-\left(E_{P}-E_{1 / 2}\right) f
$$

where $\alpha$ is the electron transfer coefficient, $E_{P}$ is the peak potential, $E_{1 / 2}$ is the half-wave potential, $f=\frac{F}{R T}$ and where $R, F$ and $T$ have their usual meanings. $\Xi(\Lambda, \alpha)$ allowed an estimation of the succeeding dimensionless parameter $\Lambda$ ([1], from graph on p. 238) proportional to the electron transfer rate constant:

$$
\Lambda=\frac{k^{0}}{\sqrt{D f v}}
$$

where $k^{0}$ is the electron transfer rate constant, $D$ is the diffusion coefficient $\left(6.7 \times 10^{-6} \mathrm{~cm}^{2} \mathrm{~s}^{-1}\right)$, $f=\frac{F}{R T}$ and $v$ is the scan rate. Values of $\Xi$ between 12.1 and 16.8 were obtained for scan rates from $5 \mathrm{mV} / \mathrm{s}$ to $150 \mathrm{mV} / \mathrm{s}$. From the Tafel plot of the anodic peak current (Fig. 4S), the transfer coefficient, $\alpha$, has been estimated to be 0.66 . Values of $\Lambda$ between $6 \times 10^{-4}$ and $3 \times 10^{-5}$ were obtained, which corresponds to a completely irreversible electrode process in accordance with the Matsuda-Ayabe criteria $\Lambda<10^{-3}$. From these values $k^{0}$ was calculated to have a value between $2 \times 10^{-7}$ and $7 \times 10^{-7} \mathrm{~cm} \mathrm{~s}^{-1}$. 


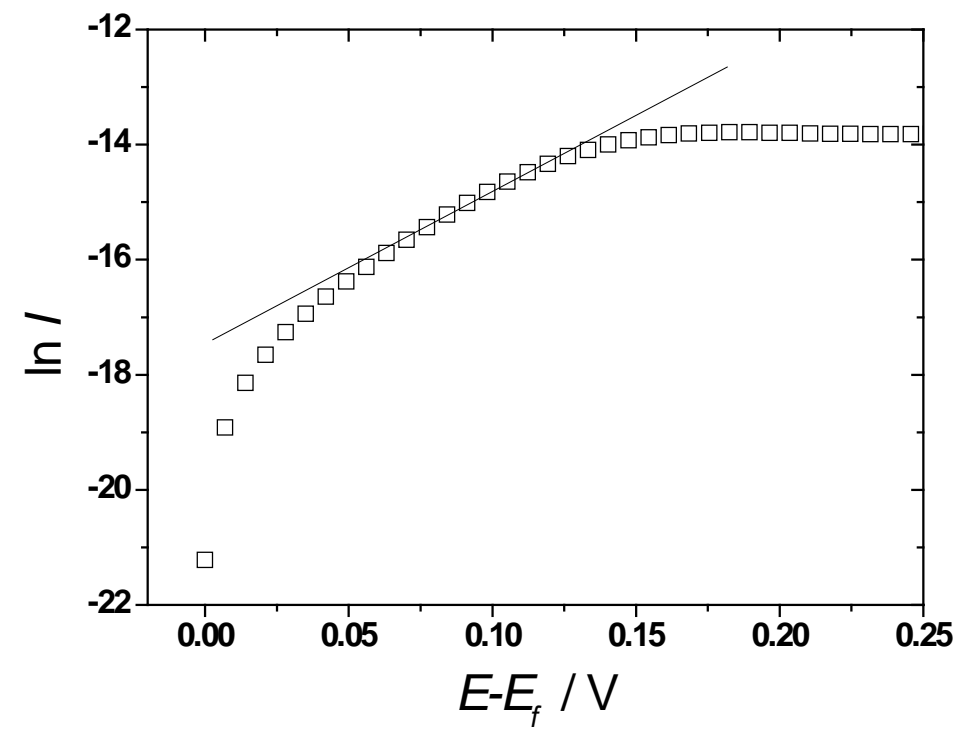

Figure 4S. Tafel plot of the anodic peak current obtained at pristine EG $(0.1 \mathrm{M} \mathrm{KCl}, 1 \mathrm{mM}$ $\mathrm{K}_{4} \mathrm{FeCN}_{6}$ ). The Tafel plot (Fig. 4S) was used for the assessment of the transfer coefficient, $\alpha$, of the electrode reaction on EG. The experimental data yields a straight line in the Tafel region with a slope of $\alpha F / R T$. The transfer coefficient was estimated to be 0.66 . 

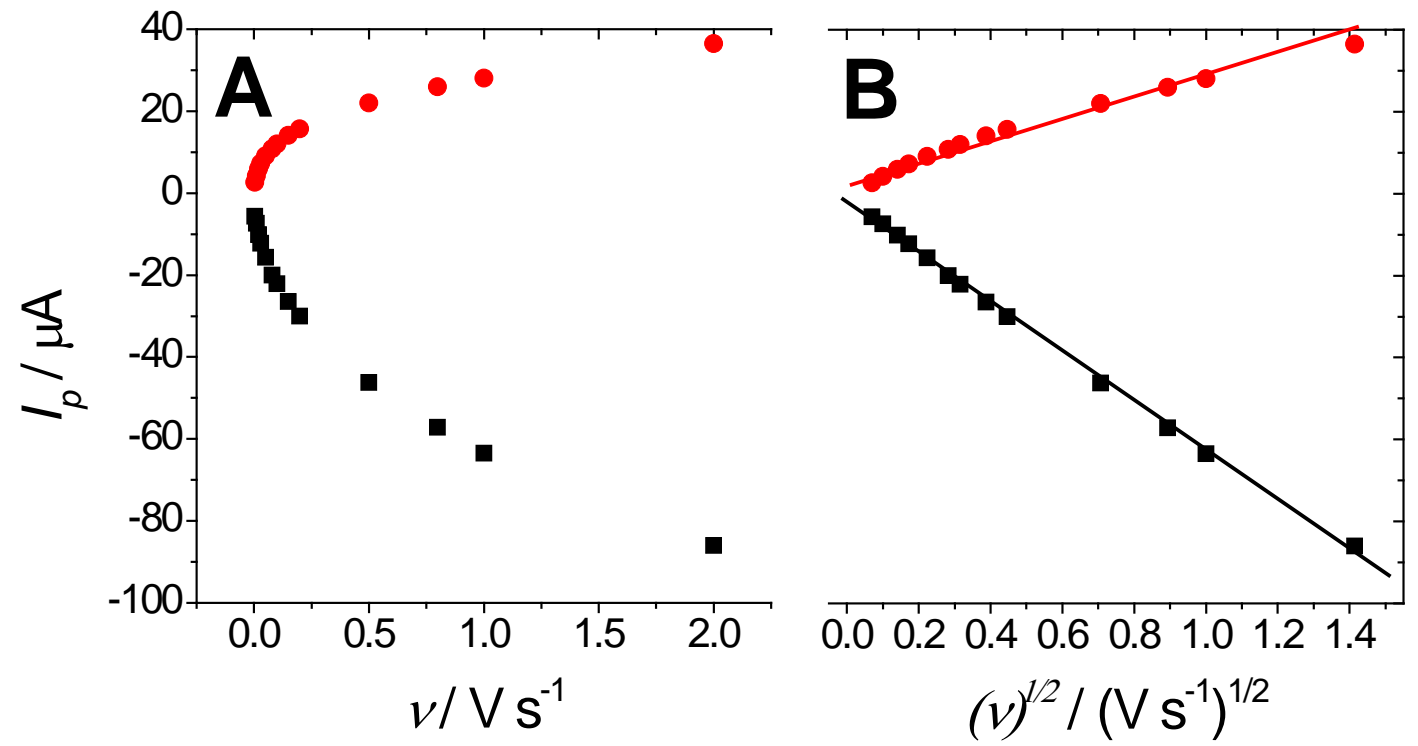

Figure 5S. Diffusion control of the hexaammine-ruthenium redox process on pristine EG. The dependencies of the anodic ( $\bullet$ ) and cathodic ( $\mathbf{})$ peak currents on the scan rate $(\mathbf{A})$ and on the square root of the scan rate (B) $\left(0.1 \mathrm{M} \mathrm{KCl,} 1 \mathrm{mM} \mathrm{Ru}(\mathrm{NH} 3)_{6} \mathrm{Cl}_{3}\right)$. 


\section{Supporting Note 2. Electron transfer rate constant estimation by the Nicholson method.}

The hexaammine-ruthenium couple is characterized by a semi-reversible redox process with peakto-peak separation potentials up to $0.2 \mathrm{~V}$. A pure diffusion control of the redox process was confirmed by a scan rate study (Fig. 5S). The slope of a plot of the $\log _{10}$ of the observed peak currents versus $\log _{10}$ of the scan rate (Fig. $6 \mathrm{~S}$ ) was 0.47 and 0.46 for the anodic and cathodic peak currents, respectively, indicating semi-infinite diffusion, which can be modelled by the RandlesSevcik equation [3]:

$I_{P}=0.4463\left(f F^{2}\right)^{1 / 2} n^{3 / 2} A D^{1 / 2} C v^{1 / 2}$

Where $n$ is the number of electrons transferred in the redox reaction, $A$ is the electrode surface area and $C$ is the concentration of the redox probe.

Thus, the Nicholson method [4] can be applied for the electron transfer rate calculation. The dimensionless parameter $\Psi$ is defined as:

$\Psi=\frac{k^{0}}{D_{O} \pi v(n f)^{1 / 2}}\left(\frac{D_{O}}{D_{r}}\right)^{\alpha / 2}$

where $D_{o}$ is the diffusion coefficient of the oxidized form of hexaammine-ruthenium $\left(D_{o}=D_{r}=\right.$ $\left.9.1 \times 10^{-6} \mathrm{~cm}^{2} \mathrm{~s}^{-1}[5]\right)$ and $\alpha$ is the transfer coefficient (0.5). From the observed peak-to-peak separations of $0.10-0.17 \mathrm{~V}$, values of $\Psi$ in the range of $0.25-0.5$ can be extracted from [4]. An estimation of the electron transfer rate constant, $k^{0}$, for the hexaammine-ruthenium redox process on pristine epitaxial graphene in the range of $0.7 \times 10^{-3}-1.2 \times 10^{-3} \mathrm{~cm} \mathrm{~s}^{-1}$ was obtained from eq. (6). 


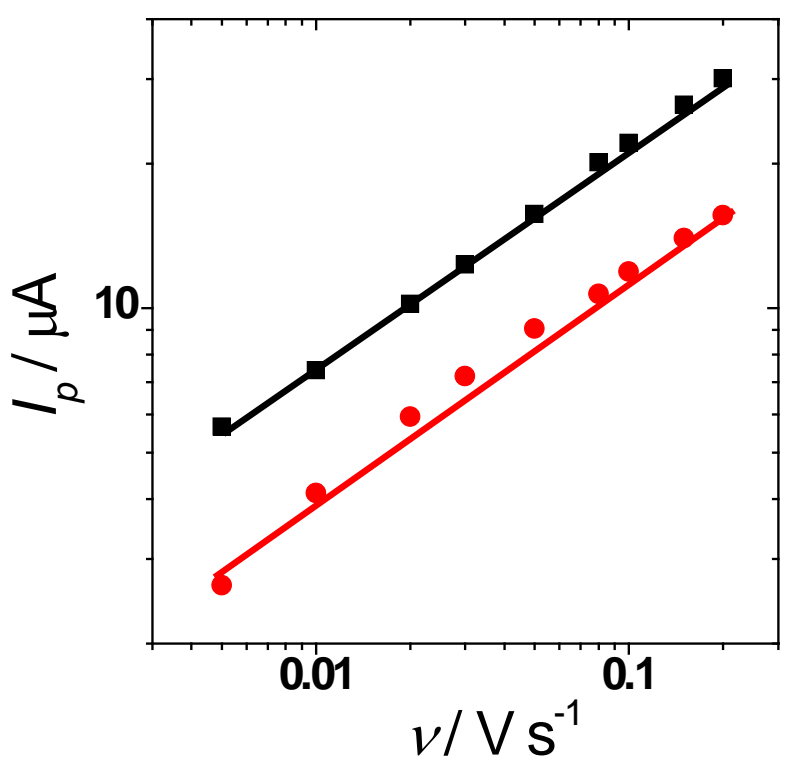

Figure 6S. The scan rate dependencies of the anodic $(\bullet)$ and cathodic $(\boldsymbol{\bullet})$ peak currents of the hexaammine-ruthenium redox process on pristine EG in double logarithmic coordinates. Slopes of the interpolated lines are close to 0.5 demonstrating semi-infinite diffusion behavior of the redox process. 

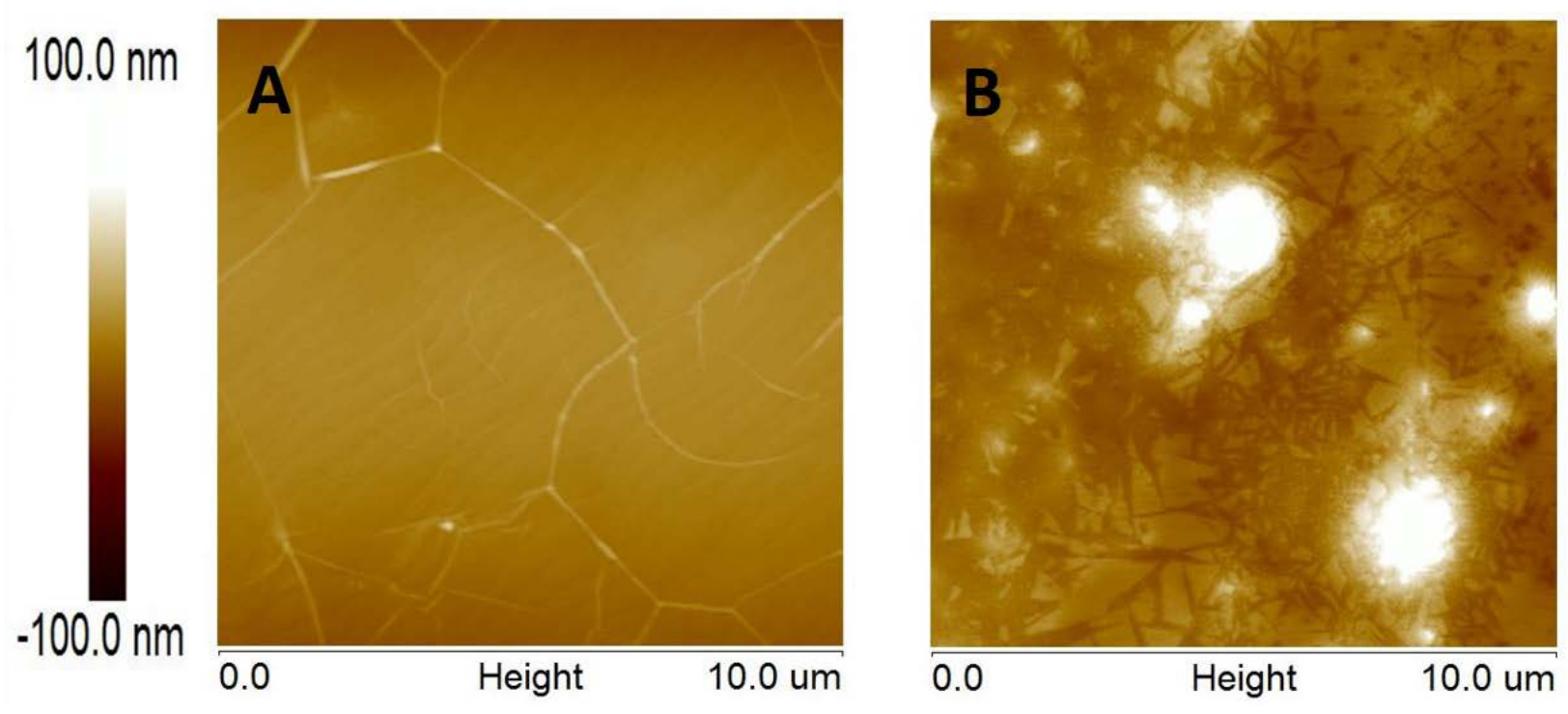

Figure 7S. AFM images of pristine (A) and anodized (B) EG. 


\section{References}

[1] A.J. Bard, L.R. Faulkner, Electrochemical Methods. Fundamentals and Applications, John Wiley and Sons, Inc, New York, 2001.

[2] H. Matsuda, Y. Ayabe, Z. Electrochem., 59 (1955) 494.

[3] R.G. Compton, C.E. Banks, Understanding Voltammetry, World Scientific Publishing Company, 2007.

[4] R.S. Nicholson, Theory and Application of Cyclic Voltammetry for Measurement of Electrode Reaction Kinetics, Analytical Chemistry, 37 (1965) 1351-1355.

[5] C.E. Banks, R.G. Compton, A.C. Fisher, L.E. Henley, The transport limited currents at insonated electrodes, Physical Chemistry Chemical Physics, 6 (2004) 3147-3152. 